\title{
Olive Oil/Soybean Oil/Multi-amino Acid/Glucose Parenteral Nutrition Emulsion
}

National Cancer Institute

\section{Source}

National Cancer Institute. Olive Oil/Soybean Oil/Multi-amino Acid/Glucose Parenteral

Nutrition Emulsion. NCl Thesaurus. Code C118366.

An electrolyte-based parenteral emulsion for infusion consisting of a three chamber bag system containing a glucose solution, an amino acid solution and a lipid emulsion that can be used to provide parenteral nutritional supplementation. The lipid emulsion portion contains 20\% refined soybean oil and $80 \%$ refined olive oil; the amino acid solution contains alanine, arginine, aspartic acid, glutamic acid, leucine, isoleucine, lysine, methionine, phenylalanine, proline, serine, threonine, tryptophan, tyrosine, and valine. The parenteral nutrition emulsion also contains the electrolytes phosphate and acetate. The three separate bags are mixed together before infusion. Upon infusion, the emulsion provides calories for energy. The lipid portion supplies essential fatty acids that can be incorporated into cell membranes. The amino acids facilitate protein synthesis. 